\title{
Untersuchungen zum Auslaugverhalten beregneter Bauteile
}

\section{Investigation of the leaching behaviour of structural elements exposed to rain}

\section{A. Vollpracht, W. Brameshuber}

\section{EINLEITUNG}

In den letzten Jahren sind zahlreiche Regelungen zur Umweltverträglichkeit von Baustoffen verschärft worden. Dieser Trend wird sich auch in den kommenden Jahren fortsetzen. Betroffen sind einerseits nationale Regelungen (z. B. die Erstellung einer Ersatzbaustoffverordnung für mineralische Abfälle und Nebenprodukte), andererseits werden im Rahmen der Arbeiten zur Harmonisierung der wesentlichen Anforderungen 03 (Hygiene, Gesundheit und Umweltschutz) der Bauproduktenrichtlinie auch in Europa Konzepte zur Bewertung der Umweltverträglichkeit erarbeitet. Kompakte Baustoffe, die oberhalb des Bodens eingebaut werden, sind derzeit noch nicht von den Regelungen betroffen. Da diese Baustoffe jedoch durch den Niederschlag ausgelaugt werden können, tragen sie ebenfalls zum Eintrag von Schadstoffen in den Boden bei. Es ist daher vorauszusehen, dass zukünftig wahrscheinlich auch beregnete Baustoffflächen bezüglich ihrer Umweltverträglichkeit geprüft werden müssen. Zu dieser Thematik liegen bisher kaum Forschungsergebnisse vor. Daher sollte in einem von mehreren Industriepartnern geförderten Projekt eine entsprechende Datengrundlage zum Auslaugverhalten beregneter Baustoffe geschaffen werden. Es wurden vier unterschiedliche mineralische Baustoffe beregnet. Parallel dazu wurde überprüft, inwieweit die Auslaugung nach dem für Beton angewendeten Langzeitstandtest /1/ mit den Ergebnissen der Beregnungsversuche vergleichbar ist.

\section{VERSUCHSDURCHFÜHRUNG}

Das Prinzip des Beregnungsversuchs ist in Bild 1 dargestellt. Es wurden Platten mit einer beregneten Fläche von $400 \cdot 300 \mathrm{~mm}^{2}$ verwendet, die auf zwei Gestellen aus Edelstahl aufgestellt wurden. Fassadenelemente wurden senkrecht stehend beregnet, Dachelemente wurden mit einer Neigung von $30^{\circ}$ eingebaut. Für die Beregnung wurden Zweistoffdüsen ausgewählt, in denen das Regenwasser durch Druckluft zerstäubt wird. Der Wasserzufluss erfolgte aus einem Tank unter Ausnutzung der Schwerkraft. Es wurde einfach deionisiertes Wasser verwendet. Bei Fassadenelementen betrug der Sprühwinkel $45^{\circ}$, Dachflächen wurden von oben beregnet. Das an der Prüfplatte ablaufende Wasser wurde in einer Auffangrinne aus PE gesammelt und in einen PE-
Behälter geleitet. Nach jedem Einzelregen wurde das Eluat entnommen und analysiert. Neben der Eluatmenge wurde auch die Wasseraufnahme der Probekörper erfasst, um die Regenmenge kontrollieren zu können. Der Versuch wurde als Doppelbestimmung durchgeführt.

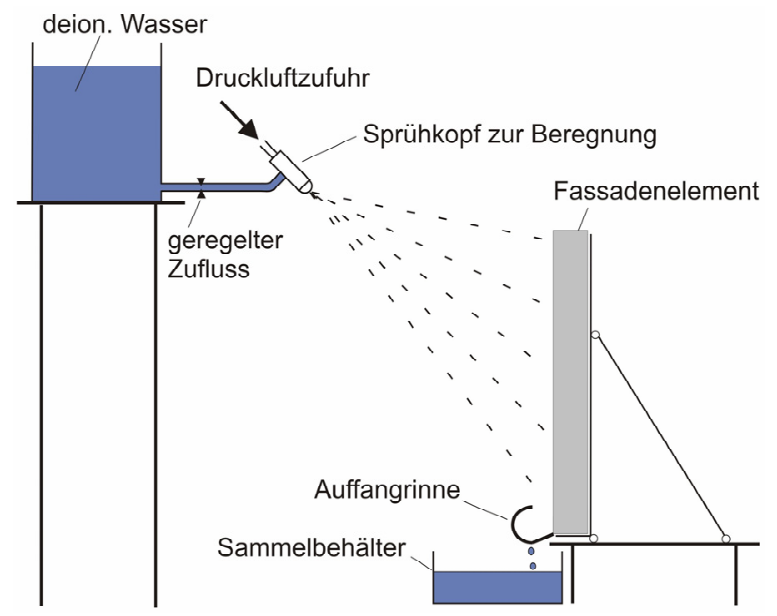

Bild 1: Schematischer Versuchsaufbau zur Beregnung von Fassadenelementen

Fig. 1: Experimental setup for the irrigation of façade elements

Die Festlegung der Beregnungszyklen erfolgte nach einer Sichtung von Wetterdaten aus ganz Deutschland. Im Rahmen von Vorversuchen wurden zunächst drei Zyklen bestimmt, die einem minimalen, einem maximalen und einem mittleren Regenmonat in Deutschland entsprechen. Beregnungsversuche mit diesen drei Testregen zeigten, dass eine direkte Korrelation zwischen der Regenmenge und der Konzentrationen an ausgelaugten Stoffen im Eluat für die meisten Parameter nicht gegeben ist. Die freigesetzte Fracht (in $\mathrm{mg}$ bezogen auf die beregnete Fläche) nimmt jedoch in aller Regel mit der Regenmenge zu. Für die Hauptversuche wurde daher der maximale Testregen für Dachflächen ausgewählt. Fassadenelemente werden nur durch Schlagregen, nicht durch Normalregen ausgelaugt, daher ist hier ein geringerer Niederschlag zu erwarten, der durch den mittleren Beregnungszyklus gut abgebildet wird. 


\section{VERSUCHSERGEBNISSE}

Anhand der Eluatanalysen und der gesammelten Regenmenge wurden die Freisetzungen in jedem einzelnen Regenereignis berechnet und auf die beregnete Fläche bezogen. Trägt man diese inkrementelle Freisetzung über der Nummer des Einzelregens auf, so sind drei grundsätzlich verschiedene Entwicklungen feststellbar. Fälle 1 und 2 sind in Bild 2 anhand unterschiedlicher Parameter dargestellt. Zum Vergleich ist auf der Sekundärachse der Niederschlag angegeben.
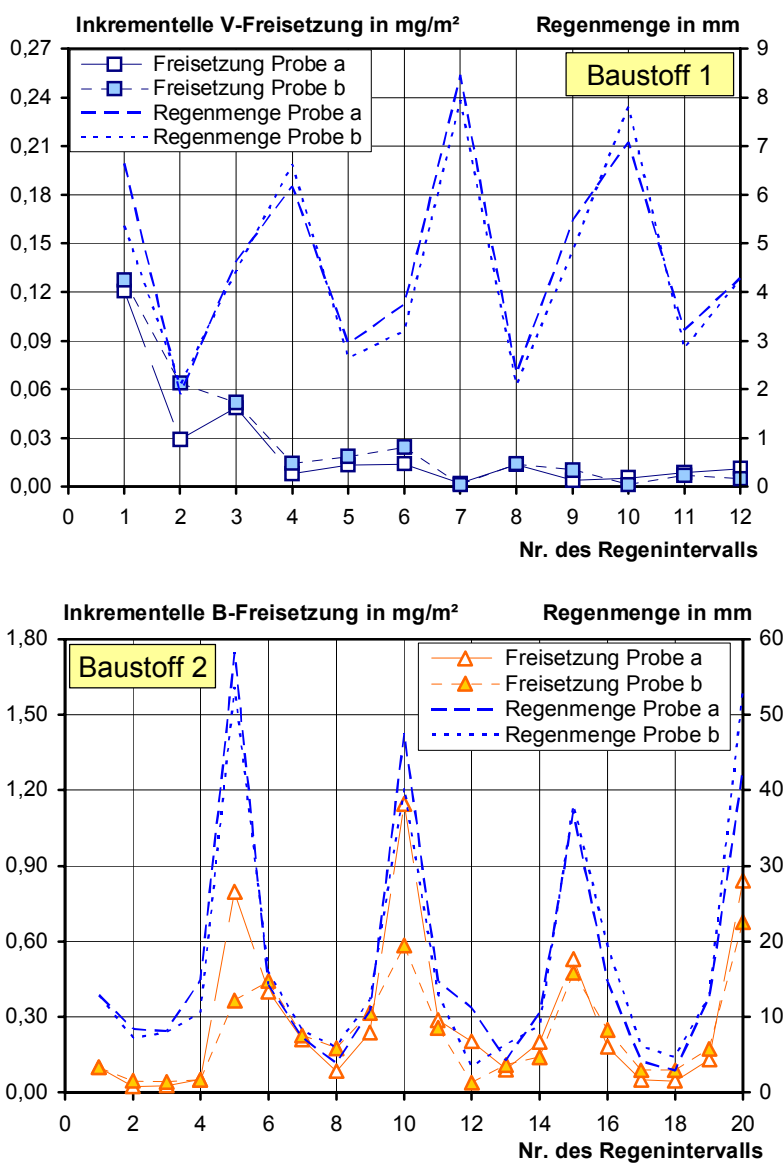

Bild 2: Inkrementelle Freisetzung im Beregnungsversuch aufgetragen über der Anzahl der Regenereignisse im Vergleich zur Regenmenge oben: Vanadiumfreisetzung aus Baustoff 1 unten: Borfreisetzung aus Baustoff 2

Fig. 2: Incremental release in the irrigation test plotted against the number of rains and compared to the amount of rain

above: release of vanadium from material 1

below: release of boron from material 2

Im oberen Bild ist die Freisetzung im ersten Eluat erhöht und fällt danach deutlich ab; die Regenmenge hat keinen signifikanten Einfluss. Im unteren Bild ist die Freisetzung dagegen proportional zur Regenmenge; eine abfallende Tendenz tritt nicht auf. Im dritten, hier nicht dargestellten Fall streuen die Kurven stärker und es ist keine funktionale Abhängigkeit der drei Parameter Freisetzung,
Regenintervallnummer und Regenmenge erkennbar. Das Auslaugverhalten der Baustoffe weist demnach grundlegende Unterschiede auf. Die Auslaugcharakteristik ist dabei sowohl vom betrachteten Parameter als auch vom Baustoff abhängig, was die Ableitung eines übergreifenden Bewertungskonzepts deutlich erschwert.

Neben den Beregnungsversuchen wurden Untersuchungen mit dem Langzeitstandtest, d. h. mit durchgehender Wasserbeaufschlagung, durchgeführt. Zum Vergleich der beiden Versuche wurden die Freisetzungen während der einzelnen Regenereignisse aufsummiert. Es zeigte sich, dass die Freisetzung im Beregnungsversuch in aller Regel deutlich unter der Freisetzung im Langzeitstandtest blieb (s. Bild 3). Nur für den Parameter Barium wurde bei einigen Baustoffen in beiden Versuchen die gleiche kumulative Freisetzung gefunden.

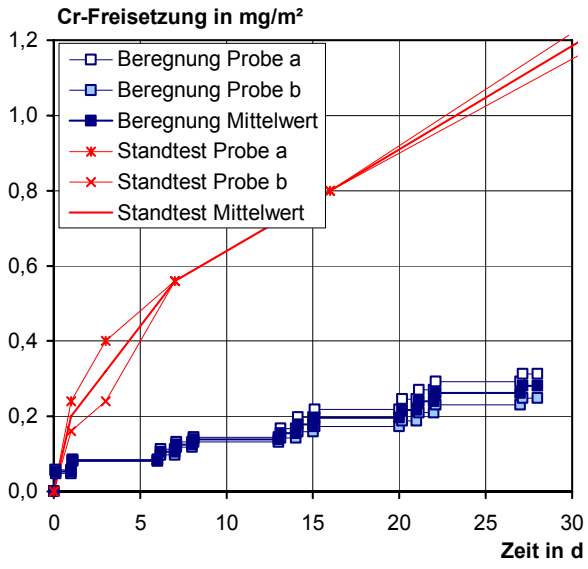

Bild 3: Freisetzung von Chrom aus Baustoff 1 im Beregnungsversuch und im Langzeitstandtest

Fig 3: Release of chromium from material 1 in the irrigation test and in the tank test

Die Auslaugung beregneter Bauteile wird also durch den Langzeitstandtest abgedeckt. Eine regelmäßige Durchführung von Beregnungsversuchen ist nicht erforderlich. Als wesentliches Ergebnis des Forschungsvorhabens ist festzuhalten, dass die meisten umweltrelevanten Parameter in den Eluaten nur in sehr geringen, nicht toxischen Konzentrationen nachweisbar waren. Die Parameter Chrom, Molybdän, Sulfat und ggf. Vanadium sollten bei einzelnen Baustoffen noch genauer untersucht werden.

\section{$4 \quad$ LITERATUR}

/1/ Deutscher Ausschuss für Stahlbeton (DAfStb): DAfStb-Richtlinie „Bestimmung der Freisetzung anorganischer Stoffe durch Auslaugung aus zementgebundenen Baustoffen“. - Ausgabe Mai 2005. Berlin : Deutscher Ausschuss für Stahlbeton

Forschungsförderer: Industrie

Kurzberichte des Institut für Bauforschung 21 (2008) Nr. 141; ISSN 1867-2019

Herausgeber:

RWTH Aachen University

Univ.-Prof. Dr.-Ing. W. Brameshuber

Univ.-Prof. Dr.-Ing. M. Raupach

Schinkelstraße 3, 52062 Aachen

Deutschland / Germany
Telefon $\quad+49(0) 24180-95100$

Telefax +49(0) $24180-92139$ www.ibac.rwth-aachen.de

datenbanken bedürfen der Genehmigung des ibac. 